\title{
Management of Jejunoileal Atresia : Our 5 year Experience
}

\section{Md. Shahjahan \\ Kazi Md. Noor-ul Ferdous* \\ Md. Ashrarur Rahman Mitul M. Kabirul Islam}

Department of Pediatric \& Neonatal Surgery Dhaka Shishu (Children) Hospital

Dhaka, Bangladesh.
${ }^{*}$ Correspondence to:

Dr. Kazi Md. Noor-ul Ferdous

Department of Pediatric \& Neonatal Surgery,

Dhaka Shishu (Children) Hospital

Dhaka, Bangladesh.

Mobile: +880-1711322487

E-mail:kmnferdous@gmail.com

\begin{abstract}
Purpose: The purpose of the study was to see the outcome of primary anastomosis and enterostomy in jejunoileal atresia, to identify the complications associated with these surgical procedures and to find out a preferable surgical option for the complicated atresia. Methods: This prospective study was carried out in the Department of pediatric Surgery, in a tertiary pediatric hospital during the period of 5 years (July 2006 to June 2011). The study included neonates of both sexes, who were per operatively diagnosed as jejuno-ileal atresia. We excluded the patients of jejunoileal atersia with associated major anomaly like complex ARM, gastroschisis, omphalocele etc. Results: During this study period, a total 176 patients were diagnosed as jejunoileal atresia. Twelve of them were excluded due to presence of major associated anomaly. So the sample size was 164 . Resection and primary anastomosis were done in 132 jejunoileal atresia with or without gangrene. Enterostomy were done in 32 cases, complicated by perforation of proximal segment with gangrene and peritoneal soiling. Mortality was higher after primary anastomosis in complicated atresia but much better than enterostomy. Complications were also more frequent and troublesome after enterostomy. Conclusion: Primary anastomosis gave better outcome in all types of jejuno-ileal atresia with or without complication which reduce the post operative morbidity and mortality.
\end{abstract}

Key words: Jejuno-ileal atresia; Primary anastomosis; Enterostomy.

\section{INTRODUCTION}

Intestinal obstruction is a common surgical emergency in the neonatal period. It occurs in approximately 1 in 1500 live birth ${ }^{1}$. Intestinal atresia is the third common cause of intestinal obstruction after Hirschsprung's disease and meconium ileus ${ }^{2}$ and is found in approximately one fifth of the patients who require major operations in the first 2 weeks of life $^{3}$. Atresia refers to a congenital obstruction caused by complete occlusion of the intestinal lumen. Atresia is common in the duodenum, jejunum and ileum but rare in the colon. In Bangladesh jejuno-ileal atresia is the most common type of intestinal atresia ${ }^{4}$. Prompt recognition of intestinal atresia is essential for adequate management to be instituted ${ }^{5,6,7}$. Bowel movement is a matter of concern if the baby fails to pass meconium for a day or two in presence of a normal looking anus. In addition to non-passage of meconium, early bilious vomiting, abdominal distension and maternal polyhydromnios is very alarming ${ }^{8}$. In developed countries, most of the congenital intestinal obstruction is being diagnosed in utero by ultrasonnogram. In Bangladesh delayed presentation of the neonatal intestinal obstruction is a common picture. Whatever the cause, delay in presentation of intestinal atresia usually associated with more complication and increased chance of morbidity and mortality ${ }^{4}$. Two most commonly practiced surgical methods for jejunoileal atresia are 'resection and end-to-back anastomosis' and early 'exteriorization or enterostomy' and subsequent end-to-back anastomosis ${ }^{9}$. Decision for surgical option in a stable case of jejunoileal atresia without any complication is towards resection and end-to-back anastomosis. The presence of gangrene and perforation of the proximal intestinal segment in jejunoileal atresia is often difficult to select better surgical option between primary anastomosis and enterostomy. Immediate postoperative outcome seems to be satisfactory after enterostomy, but subsequent care of stoma and maintenance of nutritional status is so difficult, that 
most of the survivor not able to reach the next surgical procedure of stomal closure ${ }^{10}$. Improvement in pediatric anesthesia and surgical expertise, presence of effective antibiotics and better understanding of fluid and electrolyte homeostasis; operative time and stability of the patient is a little constraint to the choice of surgical option. The present study was undertaken to compare the result of primary anastomosis and enterostomy in jejunoileal atresia and to choose a preferable surgical option, especially to those who present with complication.

\section{MATERIALS \& METHODS}

It was a prospective interventional study, done on neonates presented with jejunoileal atresia, carried out in a tertiary pediatric hospital, in the period of July, 2006 to J une, 2011 (5 years). Ethial permission was taken from the ethical committee. In each case, information about the patient was collected in a prescribed data-format after getting written consent from the parents or guardian in a preformed consent form. Data were collected by- general questionnaire, clinical examination, evaluating preoperative management, operative findings, postoperative management $\&$ follow up. Collected data were arranged in systematic manner, presented in various tables and figures and statistical analysis was made to evaluate the objectives of this study. P value $<0.05$ was taken as significant.

The study included neonates of both sexes, who were peroperatively diagnosed as jejuno-ileal atresia. We excluded the patients per-operatively revealed no intestinal atresia, duodenal atresia, colonic atresia and also jejunoileal atersia with associated major anomaly like complex ARM, gastroschisis, omphalocele etc.

Pre-operative management

A fter admission the neonates were managed conservatively and prepared for operation. Kept warm, nothing per oral, gut decompressed by both continuous and intermittent nasogastric suction with IV glucose containing half strength normal saline and antibiotics (ceftazidime and metronidazole) for covering both aerobic and anaerobic and inj. Vitamin $\mathrm{K}$ were given. Plain $X$-ray abdomen erect posture and baseline laboratory investigations including blood grouping, complete blood count, blood urea, serum creatinine and serum el ectrolytes were done. Serum bilirubin (total, direct and indirect) was measured when the baby became icteric. B efore closure of enterostomy, distal intestinal loop was irrigated with normal saline $(10 \mathrm{ml} /$ day $)$ twice daily. A ny anemia and electrolyte abnormality was corrected accordingly.

\section{0 perative management}

Under general anesthesia abdomen was explored through right supra-umbilical transverse incision. During laparotomy diagnosis of jejunoileal atresia was confirmed. A ny associated complication and anomaly was noted. Distal petency of lumen was checked to exclude additional pathology and operative treatment was planned. We grouped the included patients according to surgical procedures. Patients in group: $A$, resection and primary end to back anastomosis was done.Patients of group:A were subdivided in to Group: $A_{1}$ included jejunoileal atresia without any complication and group: $A_{2}$ complicated with gangrene of proximal dilated segment. In group: B, resection and enterostomy was done for macroscopic soiling of peritoneal cavity by perforation. Enterostomy closure was done 4-6 weeks after enterostomy.
Post operative management and follow up

Postoperatively nothing per oral, nasogastric suction, maintenance of fluid and electrolyte balance was continued. Potential nutritional supplement in the form of amino acid, albumin or blood transfusion was given in some patients. A ntibiotics were continued postoperatively for 7 days and in selective cases up to healing of the wound. Graduated oral feeding was started with evidence of progressive gastrointestinal function, such as soft flat abdomen, clear low volume gastric effluent, propulsive or stoma started to function. Initially normal saline was started and afterwards expressed breast milk was given as test feeding. If the baby tolerated this, then limited breast-feeding to feeding on demand with gradual withdrawal of intravenous fluid was ensured. A fter enterostomy closure, same principle was followed postoperatively.

A Il patients were under regular followed up in hospital, with the followings: general condition, fever, vomiting, Nesogastric suction, abdominal distention, bowel sound and bowel movement. Patients were discharged on following criteria: wound healed, on exclusive oral feeding and regular bowel movement. All the patients were advised to come after 1 week, 1 month, 3 months and 6 months. The following were observed: weight, feeding history, vomiting, bowel habit, abdominal distention, wound condition etc.

\section{RESULTS}

A total of 176 cases of jejuno-ileal atresia were admitted in DSH during the study period. Out of those, 164 were included in this study after excluded 12 cases of jejunoileal atersia with associated major anomaly like complex ARM, gastroschisis, omphalocele etc. The demographics of the study group are given in the table-1.

Table 1 : Patients' demography $(\mathrm{N}=164)$
Variables

Sex

$\begin{array}{ll}\text { M ale } & 116(70.73 \%) \\ \text { Female } & 48(29.27 \%) \\ \text { M:F ratio } & 2.4: 1 \\ \text { tional A ge } & \\ \text { Preterm } & 52(31.71 \%) \\ \text { Term } & 112(68.29 \%)\end{array}$

Weight at presentation

$\leq 2 \mathrm{~kg}$

$>2 \mathrm{~kg}$

$\mathrm{M}$ ean $\pm \mathrm{SD}$

Range

Age at presentation

$\leq 7$ days
$>7$ days
$M$ ean $\pm S D$
Range

$2.36 \pm 0.56$

$1.5-3.67 \mathrm{~kg}$

$144(87.8)$

$20(12.2)$

$4.54 \pm 3.37$

1-15 days
Resection of proximal dilated segment (with or without gangrene) and primary end-to-back anastomosis was done in 132 cases. A mong them 96 (72.73\%) survived and 36 (27.27 $\%$ ) died. In 32 patients, resection of proximal gut (gangrene with perforation, gross contamination) and enterostomy was done. 12 of them died postoperatively, 16 after discharge and before enterostomy closure and remaining 4 after enterostomy closure. Enterostomy closure was done 4-6 weeks after enterostomy.

Overall survival rate was $58.54 \%$ and mortality rate was $41.46 \%$. 
Table 2: O utcome of surgical procedures ( $n=164)$.

\begin{tabular}{|c|c|c|}
\hline Procedure & Survived (\%) & Died (\%) \\
\hline $\begin{array}{l}\text { Group A: Primary anastomosis ( } N=132) \\
A_{1}-\text { Uncomplicated ( } N=88 \text { ) } \\
A_{2} \text { - Complicated with gangrene }(N=44)\end{array}$ & $\begin{array}{l}96(72.73) \\
72(81.82 \%) \\
24(54.55 \%)\end{array}$ & $\begin{array}{l}36(27.27) \\
16(18.18 \%) \\
20(45.45 \%)\end{array}$ \\
\hline $\begin{array}{l}\text { Group B: Enterostomy for gangrene } \\
\text { with perforation ( } \mathrm{N}=32 \text { ) } \\
\text { After Enterostomy } \\
\text { After Enterostomy closure } \\
\text { Total: Group } \mathrm{A}+\mathrm{B}(\mathrm{N}=164)\end{array}$ & $\begin{array}{l}-- \\
-- \\
- \\
96(58.54)\end{array}$ & $\begin{array}{l}32(100) \\
28 \\
4 \\
68(41.46)\end{array}$ \\
\hline
\end{tabular}

In primary anastomosis group $(\mathrm{A}=\mathrm{A} 1+\mathrm{A} 2)$ - $\mathrm{A}$ nastomotic leakage were in $18.18 \%$ patients. Those were managed by relaparotomy \& converted to enterostomy; after 4-6 weeks enterostomy closed those patients were survived.

Table 3 : Overall Complications

\begin{tabular}{lcc} 
& G roup A (N=132) & Group B (N=32) \\
A nastomosis Leakage & $24(18.18 \%)$ & --- \\
Functional Obstrution & $12(9.09 \%)$ & --- \\
Persistant Jaundice $>$ 2 wks & $8(6.06 \%)$ & --- \\
Wound infecton & $8(6.06 \%)$ & $8(25 \%)$ \\
Stomal diarrhea & --- & $16(50 \%)$ \\
Peristomal excoriation & --- & $20(62.5 \%)$ \\
Weight loss & $4(3.03 \%)$ & $16(50 \%)$ \\
\hline
\end{tabular}

Total ninety six patients came for follow up regularly up to three months of discharge. All of them were in group A. Number of attended patients and their observations are shown in Table-4.

Table 4 : Follow up: number of patients and observations

\begin{tabular}{|c|c|c|}
\hline & $\begin{array}{c}\text { Group A } \\
(\mathbb{N}=132)\end{array}$ & $\begin{array}{c}\text { Group B } \\
(N=32)\end{array}$ \\
\hline \multicolumn{3}{|c|}{ After 1 week of discharge } \\
\hline Survived patient & 96 & 20 \\
\hline Bilious vomiting & 0 & 0 \\
\hline A bdominal diarrhea & 0 & 0 \\
\hline Stomal diarrhea & 0 & 16 \\
\hline Peristomal excoriation & 0 & 20 \\
\hline Weight loss & 0 & 16 \\
\hline Defaulter & 0 & 0 \\
\hline \multicolumn{3}{|c|}{ After 1 month of discharge } \\
\hline Visited patient & 96 & 4 \\
\hline Bilious vomiting & 16 & 0 \\
\hline Diarrhea & 4 & -- \\
\hline Stomal diarrhea & -- & 4 \\
\hline Peristomal excoriation & -- & 4 \\
\hline Failure to thrive & 4 & 4 \\
\hline Defaulter (?? Died) & 0 & 16 \\
\hline \multicolumn{3}{|c|}{ After 3 months of discharge } \\
\hline Visited patient & 96 & -- \\
\hline Bilious vomiting & 7 & \\
\hline Failure to thrive & 5 & \\
\hline \multicolumn{3}{|c|}{ After 6 months of discharge } \\
\hline Visited patient & 52 & \\
\hline Bilious vomiting & 3 & \\
\hline Failure to thrive & 2 & \\
\hline
\end{tabular}

\section{DISCUSSION}

J ejunal atresia is a common cause of neonatal intestinal obstraction, secondary to intra uterine mesenteric vascular accident ${ }^{11}$

The aim of the study was to identify the choice of options of the type of surgery should done in different types of jejuno ileal atresia, their outcomes and complications.

Though factors affecting the survival of patients with jejunoileal atresia are gastrational age, age at presentation, birth weight, maturity of the baby, types and site of atresia, pheumonia, septicaemia, availability of NICU and finally type of treatment offered were the variables correlated with the short term out come and survival of the patients with jejuno-ileal atresia $a^{12}$.

Majorities of our delivaries still occurred at home means that delay in presentation and surprising miss interpretation of passage of mucous as meconium by the parents and even by the primary health workers-is inevitable, which found to be a significant factors for mortality. Delay prior to admission was the common observation found in this study, which lead to demise of many patients due to septicaemia, aspiration pneumonia and metabolic disturbance. Majority of these patients presented after the 4 days of life during which time patients could have vomiting, developing pneumonia, systemic sepsis and metabolic disturbance, due to obstracted bowel ${ }^{13}$.

The presence of associated complication affect the survival significantly such as meconium peritonitis, hypertrophioc proximal gut and others. The types of atresia and operative procedure affected the outcome as all these patients were treated in similar circumstances. A pple-peel (Type-IIIB), multiplea atresia (Type-IV) with entarostomy was the worst prognosis and higher mortality than other types. J ejuna atresias are of more severe form than ileal atresia ${ }^{12}$. Early diagnosis and prompty treatment of these patients has been stressed upon by many observers ${ }^{2}, 9,14$.

The longer the time taken to present to the hospital, for diagnosis, resacitation and surgical intervention was, the graver the prognosis would be. Mortality was also found to be associated with location and types of atresia. The more distal the lesion, the more are the chances of survival ${ }^{12}$.

Majority of our study population was admitted into hospital within first week of life. Jafor ${ }^{4}$ also observed that majority of jejuno-ileal atresia patients admitted within 7 days of life. Early diagnosis and treatment of this absolutely surgical condition has a great impact on metabolic and respiratory reserve of these small patients as well as subsequent outcome ${ }^{15}$.

$M$ ajority of neonates the study were full term. Jafor ${ }^{4}$ also reported that majority of his patients was term and Nixon and Tawes $^{16}$ stated that maturity was an important prognostic factor in intestinal atresia. A deyemi ${ }^{5}$ reported that gestational maturity did not appear to influence aurvival significantly.

Our study shown that the weight at admission the most of the neonates with atresia were more than $2 \mathrm{~kg}$. This grouping was based on use of "risk" group by Nixon and Tawes ${ }^{16}$. Smith and Glasson $^{15}$ (1989) reported a mean birth weight of $2.77 \mathrm{~kg}$ and Chang et $\mathrm{al}^{8}$ observed weight at admission which was not representative of birth weight. A deyeni ${ }^{5}$ also stated that weight was not significantly influence survival.

Outcome of surgical treatment after primary anastomosis in jejunoileal atresia without complication (Group A ) was better, in our study. M ortality in complicated group was high both in 
primary anastomosis (Group $A_{2}$ ) and enterostomy (Group B), but outcome was worse in later one. Postoperative complication was less after primary anastomosis and its management was used to be less troublesome.

Survival of the neonates with jejunoileal atresia has improved from $64 \%$ to $90 \%$ in most of the series ${ }^{6,9}, 10,16,17$.

Probably the most important factor in the improved outlook for infants with jejunnoileal atresia was the ability of adequate support in improving the nutritional status of these patients with total parenteral nutrition and highly defined elemental diets ${ }^{9,18}$. In our study with an overall operative survival rate of jejunoileal atresia was $58.54 \%$, without adequate neonatal intensive care facilities and total parenteral nutrition.

Postoperatively complications were anastomotic leakage, peristomal excoriation, failure to thrive and/ weight loss, wound infection, stomal diarrhea, persistent jaundice and functional obstruction. Postoperative complications were less frequent in group A (Table-4). A Imoutaz A. Eltayeb and Ozturk $\mathrm{H}$ et al also observed same types of complications ${ }^{19,20}$. Though immediate postoperative outcome after enterostomy seems to be satisfactory, but loss of large amount of effluent makes maintenance of stomal care difficult and failure to thrive postoperatively became a common problem ${ }^{10}$. M any authors have suggested that primary bowel anastomosis should be attempted in all cases as temporary enterostomies have resulted into prolonged morbity and raised mortality of these patients $2,9,21$.

Present trend of enterostomy, in intestinal atresia was justified as patient in this complicated group used to be more unstable and enterostomy needed less operative time. Improvement in pediatric surgical anesthesia and better understanding of neonatal care especially in pediatric surgical setup, stability and operative time is a little constraint to choose a surgical procedure. Parenteral nutrition is an important factor, in improving survival of intestinal atresia after primary anastomosis in most of the recent series ${ }^{2,12,22}$.

All patients did not come for regular follow up. It was observed that parents came for follow up only when they face any illness, like bilious vomiting and failure to thrive, which were managed conservatively.

\section{CONCLUSION}

Primary end-to-back anastomosis gives better outcome in all types of jejunoileal atresia with or without complications. Whatever the treatment options of different type of intestinal atrasia prognosis changed in developed and developing countries. In our developing country prognosis is still poor due to late presentation and diagnosis, lack of perenteral nutritional support and inadequate neonatal intensive care support.

\section{REFERENCES}

1. Wyllie, R. 'Intestinal atresia, stenosis and malrotation', in Nelson textbook of Pediatrics, $17^{\text {th }}$ ed., B ehrman, R.E., K liegmen, R.M . and J enson, H.B., Saunders, Philadel phia, 2004;47:1232-36.

2. Touloukian, R.J. 'Diagnosis and treatment of jejunoileal atresia', World J Surg, 1993; 17: 310-17.

3. Spencer R. The various patterns of intestinal atresia. Surgery. $1968 ; 64(3): 661-8$.

4. Jafor, M.A. 'Congenital intestinal atresia and stenosis: management and outcome', M S thesis, U niversity of Dhaka, 2000; 4-80.

5. A deyemi, D. 'N eonatal intestinal obstruction in a developing tropical country: patterns, problems and prognosis', J Trop Pediatr, 1989; 35: 66-70.

6. De Lorimier, A.A., Fonkalsrue, E.W. and Hays, D.M .'Congenital atresia and stenosis of the jejunum and ileum', Surgery, 1969;65: 819-27.

7. Grosfeld, J.L.' J ejunoileal atresia and dtenosis', in Pediatric Surgery, $5^{\text {th }}$ Ed., O' N eill, J.A ., R owe, M .I., Grosfeld, M .I., Fonkalsrud, E.W., and Coran, A.G. M osby, St Louis, 1998; 47: 1145-58.

8. Chang, W.T., Chen, H.C. and Peng, H. C. 'J ejunoileal atresia in neonates', Chin M ad J (Taipei), 1995; 56: 36-38.

9. Rescorla, F.J. and Grosfeld, J.L.'Intestinal atresia and stenosis: analysis of survival in 120 cases', Surgery, 1985; 98: 668-76.

10. Prasad, T.K.S. and Bajpai, M. 'Intestinal atresia', Ind J Pediatr, 2000; 67: 671-78.

11. L ouw JH, Barnard CN, Congenital Intestinal A trsia: Observation on it's origin. Lancet, 1955; 269:490-1

12. Shakya V C, A grawal CS, Shrestha P, Poudel P, K hanya S, A dhikary S. M anagement of jejunoileal atrasias; an experience at estern Nepal. BioM ed Central Surgery 2010; 10:35. doi:10.1186/1471-2482-10-35.

13. K han K, Hazratullah, Rehman F. M anagement of patients with jejunoileal atresia:Factors affecting survival. J ournal of Postgraduate M edical Institute. 1997;11(2):192-99.

14. A Deyemi D, Neonatal Intestinal Obstraction in a developing tropical country: patern, Problem and Prognosis. J Trop Pediatric. 1989; 35(2):66

15. Smith GH , Glasson M. Intestinal atresia. A us N J Surg. 1989; 2: 599: 151

16. Nixon HH, Tawes R. Etiology and treatment of small intestine atresia: analysis of a comparison with 62 deodenal atresia. Surgery; 1971;69:41.

17. M artin LW, Zerella JT. J ejunoileal atresa: Proposed classification. J ournal of pediatric Surgery, 1976; 11;399-403

18. Barrack SM , Kyambi J M, N dungu I, Wachira N,A nangwe G, Safwat S. Intestinal A tresia and stenosis as seen and treated at $K$ enyatta national Hospital, Nairobi, E A frican M ed J ournal, 1993:70:558-64.

19. A Imoutaz A. Eltayeb. Different surgical techniques in management of small intestinal atresia in high risk neonates. A nnals of Pediatric Surgery, 2009; 5(1): 31-35

20. Ozturk H, Ozturk H, Gedik S, Duran H, Onen A. A comprehensive analysis of 51 Neonates with congenital intestinal A tresia, Saudi Med J ournal; 2007; 28(7): 1050-54.

21. Powell RW, Stapled Intestinal A nastomosis in neonates and infant; use of the endoscopic intestinal stepler, J ournal of pediatric surgery, 1995; 30(2)2:195.

22. M anning C, Streuss A, Gyepes M T. J ejunal A tresia with apple peel deformity: A report of 8 survivers, journal of Perinatal, 1989; 9(3):281. 